\title{
Féeries
}

Études sur le conte merveilleux, XVII ${ }^{\mathrm{e}} \mathrm{XIX} \mathrm{X}^{\mathrm{e}}$ siècle

\section{Les études sur le conte merveilleux en Russie}

Tradition orale et conte littéraire ( $\mathrm{XIX}^{\mathrm{e}}-\mathrm{XXI}^{\mathrm{e}}$ siècle)

The Fairy Tale Studies in Russia: the Oral Tradition and the Literary Fairy Tale (19th-21 $1^{\text {st }}$ centuries)

\section{Marina Guister}

\section{(2) OpenEdition Journals}

Édition électronique

URL : http://journals.openedition.org/feeries/715

DOI : $10.4000 /$ feeries. 715

ISSN : 1957-7753

Éditeur

UGA Éditions/Université Grenoble Alpes

Édition imprimée

Date de publication : 1 juillet 2009

Pagination : 225-240

ISBN : 978-2-84310-140-3

ISSN : $1766-2842$

\section{Référence électronique}

Marina Guister, «Les études sur le conte merveilleux en Russie », Féeries [En ligne], 6 | 2009, mis en ligne le 15 septembre 2010, consulté le 08 septembre 2020. URL : http://journals.openedition.org/ feeries/715; DOI : https://doi.org/10.4000/feeries.715 


\title{
Marina Guister
}

Institut de linguistique, RGGU, Moscou

\section{LES ÉTUDES SUR LE CONTE MERVEILLEUX EN RUSSIE}

\author{
TRADITION ORALE ET CONTE LITTÉRAIRE (XIX ${ }^{\mathrm{e}}$-XXI ${ }^{\mathrm{e}}$ SIĖCLE)*
}

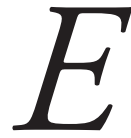

N I766-I768, LE PREMIER RECUEIL de contes littéraires en Russie, Le Persifleur ("Peresmechnik ») de Tchoulkov, prédécesseur de Gogol et de Saltykove-Schedrin, voit le jour. Cependant, ce genre littéraire, a fortiori ses premiers exemples, les ouvrages des auteurs des années $1760-1800$, est un domaine très peu étudié en Russie jusqu'à aujourd'hui, et encore moins ailleurs où ce champ littéraire paraît une terra incognita. Il s'agira dans cet article de brosser un panorama des principales études consacrées au genre du conte en Russie, qu'il s'agisse du conte littéraire ou du conte populaire, car les études les plus sérieuses sur ce genre sont souvent le fait, en Russie, de spécialistes du conte folklorique, ce qui n'a rien d'étonnant dans la mesure où l'emprunt d'idées, de structures narratives, ou même de sujets au conte folklorique est très fréquent dans la littérature, sans parler de la proximité typologique entre textes narratifs oraux et littéraires et ce qu'on appelle souvent les "sujets éternels " ou les "sujets errants ». Nous allons aborder dans cet article les étapes principales de l'étude du conte en Russie - sachant que l'approche scientifique de ce genre du folklore, comme de tous les autres genres de la littérature orale, ne sera inaugurée qu'au milieu de $\mathrm{XIX}^{\mathrm{e}}$ siècle. Nous parlerons des ouvrages concernant aussi bien l'histoire que la structure du conte, littéraire ou orale. Au niveau des études structurales, nous toucherons aux ouvrages de Propp qui sont bien connus en Europe, mais nous envisagerons également les travaux d'A. I. Nikiforov dont les découvertes, très proches de celles de Propp et contemporaines de celles-ci, sont peu connues ailleurs qu'en

* J'ai honneur de remercier la Fondation Maison des sciences de l'homme à Paris de m'avoir accordé la bourse Diderot (responsable scientifique : Maurice Aymard ; gestionnaire du programme : Annamaria Bosc) dont j'ai joui en janvier - juillet 2008. C'est pendant cette période que le présent article fut conçu. 
Russie. Nous présenterons finalement les études de l'école structuraliste russe, depuis les ouvrages de E. M. Mélétinski et de ses collègues parus aux années 1970, jusqu'aux travaux des chercheurs actuels.

Les premiers recueils de contes littéraires en Russie (ceux de M.D. Tchulkov, de M.I. Popov, de V.A. Levchine et beaucoup d'autres), rassemblent les ouvrages originaux des auteurs, les textes traduits de la littérature occidentale, les adaptations libres des bylina (chansons épiques ou ballades héroïques dans le folklore russe), les répliques enjolivées des contes et des romans d'aventures dont les sujets remontent aux livres de colportage, et les versions des contes oraux que leurs auteurs récitent de mémoire, les ayant appris directement des conteurs ou puisant aux premiers manuscrits et publications de ces textes datant des XVII ${ }^{\mathrm{e}}$ XVIII $^{\mathrm{e}}$ siècles. M. D. Tchulkov, M. I. Popov et V. A. Levchine n'étaient pas seulement les premiers auteurs à pratiquer le conte littéraire en Russie, ils étaient aussi les premiers folkloristes.

À M.Tchulkov, on doit le Recueil de chants populaires (St-Pétersbourg, I770-I774), le Dictionnaire des superstitions russes (I786), l'ABC des superstitions, des sacrifices païens, et des coutumes de mariage, en Russie ("Abevega Russkih soueverij "), et le Recueil de chants slaves (I787). V. Levchine consacre une grande partie de son recueil Les Contes Russes ("Russkije skazki ») aux bogatyr's (les héros) des bylina, où il conserve le sujet originel des textes de la poésie populaire, tout en y ajoutant des traits du roman d'aventures et du conte merveilleux. Ses versions ne sont pas toujours fidèles, mais le sujet est au moins reconnaissable. Enfin, M. Popov a recueilli les chansons populaires russes et a produit, à son tour, un livre de romans-contes ${ }^{\mathrm{I}}$ de sujet russe ou quasi russe, sous le titre Les Antiquités slaves, ou les aventures des Princes Slaves.

Les premiers auteurs des contes littéraires furent aussi les premiers à rédiger des réflexions sur ce genre littéraire, dans les préfaces de leurs recueils. Ainsi, selon M. Tchulkov, le roman ou le conte sert uniquement à divertir et non pas à instruire ou à corriger les mœurs. Quatorze ans plus tard, dans l'avant-propos de ces Contes Russes, V. Levchine propose une thèse opposée :

Tous les peuples eurent leurs romans et leurs contes. Ceux-ci nous ont laissé des descriptions très fidèles des us et coutumes de chaque pays, et ont mérité d'être fixés par écrit ; et à l'époque moderne, les peuples les plus éclairés leur ont fait honneur, en

I. Terme proposé par V. Sipovskij pour les premiers recueils de contes littéraires russes, voir ci-dessous. 
les recueillant et en les mettant sous presse. Les histoires de chevaliers contenues dans la Bibliothèque universelle des romans, de Paris, ne sont rien d'autre que des contes de bogatyr's, et la Bibliothèque bleue française contient les mêmes contes que ceux qui sont contés chez nous, dans des milieux populaires simples. Dès 1778 à Berlin, on imprime de même manière la Bibliothèque des romans, en deux parties : romans de la chevalerie allemande de jadis, et romans populaires. La Russie a, elle aussi, ses romans, or ceux-ci sont conservés seulement dans la mémoire. J'ai décidé de suivre l'exemple des éditeurs qui I775-1789 ; ainsi publié-je les Contes Russes, afin de sauvegarder ce domaine de nos antiquités ${ }^{2}$.

De cette manière, Levchine revendique le rôle éducatif et historique de son ouvrage. Précisons cependant que ce dernier est bien loin des textes folkloriques, et que sa démarche n'a pas la portée de la "découverte » du conte folklorique telle qu'on la trouve, un peu plus tard, en Allemagne, dans l'ouvrage des frères Grimm.

Les premiers recueils de contes, qu'ils soient anonymes ou rédigés par des écrivains connus, sont en général destinés aux lecteurs des milieux les plus simples de la société, et sont méprisés par l'élite. De la même façon sont accueillis les contes de loubok $k^{3}$, ou les contes de colportage, analogues russes des livres et des images d'Épinal, puisant leurs sujets tantôt dans l'oralité, tantôt dans les recueils de contes littéraires du XVIII ${ }^{\mathrm{e}}$ siècle ou bien encore dans les traductions de romans de chevalerie. Ainsi le conte extrêmement populaire du Prince Bova (Bova-korolevič) est en fait une version russe du roman anglo-normand Beufves de Hantonne. Cette dernière tradition du loubok attire l'attention d'un collectionneur pénétrant, D. A. Rovinskij, dont la collection de Tableaux populaires russes est publiée en I88I, en 9 volumes, assortie de commentaires riches mais parfois naïfs. Le genre «loubok» reste néanmoins pratiquement ignoré par les spécialistes, à la seule exception de V. D. Kuz'mina dont la monographie parue en 1964 porte sur le roman de chevalerie en Russie ${ }^{4}$. La citation suivante de N.I. Novikov (écrivain, éditeur de plusieurs revues satiriques et didactiques, directeur de l'imprimerie universitaire de Moscou et figure importante de la franc-maçonnerie et des Lumières russes) est révélatrice de cette vision élitaire du conte. Dans un article de sa revue Zivopisets ( Le Peintre »), il écrit en 1779 :

2. V. A. Levchine, Rousskije skazki, Moscou, I780. Je traduis.

3. "Loubok" est un terme standard utilisé en Russie pour la littérature de colportage. Étymologiquement il remonte à "loub»- espece du bois, et correspond à deux choses : I. planche à lithographie ; 2. éventaire du colporteur.

4. V. D. Kouz'mina, Rytsarskij roman na Roussi, Moscou, 1964. 
En France, qui saurait croire que les contes merveilleux soient mieux diffusés que les ouvrages de Racine ? Or c'est ce qui se passe chez nous : on a vendu bien plus de Mille et une nuits que d'ouvrages de Monsieur Soumarokov... O tempora, o mores! Courage, les auteurs russes! On va bientôt cesser d'acheter vos œuvres !

Or il est lui-même le premier éditeur des contes de Levchine, aussi bien ceux issus de sa plume que ceux traduits par ce dernier du français, contes orientaux y compris. Cette exception s'explique sans doute autant par leur amitié maçonnique que par des raisons commerciales. L'attitude de Novikov est en tout cas typique de l'époque : de la fin du XviII ${ }^{\mathrm{e}}$ jusqu'aux années vingt du $\mathrm{XIX}^{\mathrm{e}}$ siècle, on constate que les écrivains russes les plus remarquables sont des lecteurs assidus de contes. C'est notamment le cas d'A. N. Radischev, et ensuite d'A. S. Pouchkine, qui écrivent chacun un poème sous titré Bova, dont le sujet est emprunté aux contes loubok de Bova-korolevič (voir supra), mais dont le style et le ton évoquent La Pucelle d'Orléans de Voltaire, auquel tous les deux rendent hommage dans leurs textes.

C'est aussi au XIX ${ }^{\mathrm{e}}$ siècle que les folkloristes russes commencent à rassembler et à recueillir les textes de la tradition orale - ceux des contes, des légendes, des bylina, des chansons populaires. Vers la fin du siècle, la fidélité de ces recueils va croissant. En I855-I864 voient successivement le jour les huit volumes de la première édition de Contes populaires russes de A.N. Afanasiev : la cinquième édition de cet ouvrage, préparée, coordonnée et commentée par M. K. Azadovskij, N. P. Andreev et Ju. M. Sokolov, paraît en 3 volumes en 1936-1940. Elle fera autorité dans le monde entier et sera en général élue par les spécialistes pour sa grande précision textologique et analytique. Afanasiev figure parmi les premiers folkloristes qui se proposent l'objectif de publier les narrations populaires telles qu'elles furent présentées par les conteurs du peuple, sans les corriger et les enjoliver d'une mise en forme littéraire.

À l'époque où est publiée la collection des contes oraux d'Afanasiev, paraît la première version littéraire du conte de La Belle et la Bête (contetype 425 A, B et C) : il s'agit du conte La Fleurette Rouge ("Alen'kij tsvetochek », I856-I857), de Serguei Axakov. L'auteur fait passer son ouvrage pour un conte populaire qui lui a été raconté lorsqu'il était enfant par une certaine paysanne Pélaguée, intendante de son grand père. Il avoue également qu'en lisant plus tard le Magasin des enfants de $\mathrm{M}^{\mathrm{me}}$ Leprince de Beaumont, il avait reconnu ce même conte dans La Belle et la Bête. Une chose est claire : Axakov doit le sujet de son conte à la fois à $\mathrm{M}^{\mathrm{me}}$ Leprince de Beaumont (son Magasin avait paru cent ans avant La Fleurette Rouge et 
les Contes d'Afanasiev) et à la tradition du conte populaire russe, transmise par l'intermédiaire d'Afanasiev ou de l'intendante Pélaguée.

Après Afanasiev, des enregistrements de plus en plus fidèles de contes oraux paraissent en grande quantité, ce qui aboutit, en I9II, à la création d'une "Commission du Conte" au sein de la Société géographique russe. N'oublions pas que jusqu'aux années i9ıo, coexistent deux traditions, celle du conte oral encore vivante et celle du conte loubok. Mais ces années sont également marquées par la parution de deux ouvrages consacrés totalement ou en partie aux premiers contes littéraires russes et à leurs auteurs. En I9IO, dans ses Essais d'histoire du roman russes, V.V. Sipovskij propose une classification raisonnée de romans-contes de Tculkov, Popov et d'autres auteurs des premiers contes de la littérature russe, en les résumant de manière détaillée et en mentionnant leurs sources possibles - essentiellement des œuvres de la littérature européenne occidentale. L'étude des sources folkloriques et littéraires du conte littéraire russe du $\mathrm{XVIII}^{\mathrm{e}}$ siècle fut entreprise au I9I4 dans l'ouvrage de S. V. Savtchenko : Le conte populaire russe : histoire de sa collecte et de son étude 6 . V.V. Sipovskij et $S$. V. Savtchenko attribuèrent faussement les contes de V. Levchine à M. Tchulkov; cette erreur sera corrigée par V. B. Chklovski dans son livre Tchulkov et Levchine (Moscou, 1933).

Après la parution, en I859, du recueil de contes indiens du Panchatantra, publiés et commentés par Théodore Benfey, la proximité des sujets thématiques des contes attire l'intérêt d'un grand nombre de spécialistes. En I9IO, paraît la classification générale des contes-types - le catalogue d'Antti Aarne, traduit ensuite en anglais et puis complété par Stith Thompson. En 1929, le catalogue des sujets de contes selon le système d'Aarne, élaboré par N. P. Andreev est publié par la Société géographique. Mais avant le travail d'Andreev, était paru, en I9II-I9I4, l'ouvrage de A. M. Smirnov, Index systématique des thèmes et des variantes des contes populaires russes, dont la proximité avec le catalogue d'Aarne est impressionnante ${ }^{7}$.

En 1924, la Commission du conte de la Société géographique est rétablie sous la direction de S. F. Oldenbourg ${ }^{8}$. Dans sa revue annuelle voient le jour des articles portant sur les matériaux propres des contes régionaux,

5. V. V. Sipovskij, Otcherki iy istorii rousskogo romana, St-Pétersbourg, I9IO, vol. I et 2.

6. S. V. Savtchenko, Rousskaja narodnaja skazka: Istorija sobiranija i izoutchenija, Kiev, I9I4.

7. Pour information sur les classifications des contes russes, nous invitons à se référer à l'article panoramique de Galina Kabakova paru en 2005 dans le numéro 57-58 des Cahiers de littérature orale: «Nommer/Classer les contes populaires".

8. Voir les actes de la Commission du conte Skazotchnaja komissija v 1926, Leningrad, 1927 et Skazotchnaja komissija $v$ 1927, Leningrad, 1928. 
ainsi que des travaux consacrés au genre du conte en général. Dans ces deux domaines paraissent les ouvrages remarquables de A.I. Nikiforov' ${ }^{9}$ spécialiste du conte merveilleux du nord de la Russie et des schémas narratifs des contes, qui étudie avec beaucoup de précision et de profondeur la proximité des sujets thématiques des contes dans différentes traditions orales. C'est aussi lui qui, en même temps que Propp sinon un peu avant lui, inaugure l'étude structurelle et morphologique du conte ${ }^{\mathrm{I}}$. Nikiforov étudie " la loi de la formulation catégorielle ou grammaticale de l'action ", et il parle des "actions narratives" en termes de morphologie, en distinguant ainsi les actions "préfixales ", "radicales ", "suffixales » et "flexionnelles "; il propose enfin son schéma des personnages dont la proximité avec le "modèle structural des actants ", d' A. J. Greimas, est soulignée par E. Mélétinski dans son article : «L'étude structurale et typologique du conte" (voir infra).

Dans le recueil d'ouvrages de la Commission du conte de l'année 1926 (paru en 1927) voit le jour l'article " Morphologie du conte merveilleux russe $^{\mathrm{II}}$ » de V.J. Propp, anticipant sur son ouvrage classique Morphologie $d u$ conte $^{12}$ qui allait paraître l'année suivante. Dans ce dernier, Propp propose pour l'analyse des structures narratives du conte merveilleux un schéma qui prétend être universel et qui, en effet, fonctionne pour la majorité des contes merveilleux (et qui est applicable également au texte littéraire, bien que cette application ne soit pas conçue par l'auteur de l'ouvrage). À certaines exceptions près, la Morphologie de Propp fournit un ensemble d'outils suffisant pour décrire tout texte appartenant au genre du conte merveilleux. Cette étude en synchronie serait pourtant incomplète si les mêmes matériaux n'étaient aussi abordés sous un angle diachronique, ce que Propp entreprend dans son autre ouvrage fondateur Les Racines historiques du conte merveilleux $x^{13}$, où il propose de voir dans le conte une description de rite initiatique dont l'interprétation se modifie

9. La publication de sa sélection de contes avec commentaires est à paraitre cette année à Moscou, éditions OGI.

IO. A. I. Nikiforov, "K voprosu o morfologitcheskom izutchenii narodnoj sk azki ", Sbornik statej v chest' akademika A. I. Sobolevskogo, Leningrad, 1928, p. I72-178. L'article est écrit deux ans avant sa publication.

II. V. Ja. Propp, "Morfologija russkij volchebnoy skazki ", dans Skazotchnaja komissija v 1926, Leningrad, 1927, p. 48-49.

I2. Dans sa réponse à Claude Levi-Strauss (voir infra) Propp va blâmer le premier éditeur de Morphologie du conte pour avoir supprimé le mot " merveilleux " du titre de son livre, comme si son système de fonctions s'appliquait à toutes les espèces génériques du conte, et non pas au seul conte merveilleux.

I3. V. Ja. Propp, Les Racines historiques du conte merveilleux, Paris, Gallimard, I983. 
au fur et à mesure de l'évolution sociale. Remarquons encore qu'avant la publication des Racines historiques (mais au moment où Propp est déjà en train de travailler à cet ouvrage), paraît en 1932 dans le volume VIII de l'édition La langue et la littérature ${ }^{14}$, l'article de S. J. Lourier, "Maison dans la forêt ", portant sur le même sujet que le chapitre IV des Racines historiques - la Grande maison, notamment à propos des maisons initiatiques cachées dans la forêt et de leurs occurrences dans les contes. Lourier souligne la proximité thématique de contes relevant de sujets différents, et il remarque également la présence d'éléments communs dans des contes appartenant à des types ${ }^{15}$ différents ${ }^{16}$, c'est-à-dire de motifs qui seront un peu plus tard étudiés et catalogués par Stith Thompson ${ }^{17}$.

Après sa publication en anglais, en 1958, puis en français, en 1960, la Morphologie du conte de Propp, on le sait, est bien accueillie par les spécialistes de la littérature et du folklore et par les structuralistes en France et en Europe. Claude Levi-Strauss a consacré à ce livre son article bien connu : "La structure et la forme. Réflexions sur un ouvrage de Vladimir $\operatorname{Propp}^{18} »-$ article essentiellement favorable bien que polémique, mais qui fut cependant fort mal reçu par l'auteur de la Morphologie. Propp répondit à Levi-Strauss, dans son article "L'étude structurelle et historique du conte merveilleux ${ }^{19}$ ", de manière très agressive et en termes de duelliste, écrivant par exemple : "Pour un homme agressé, il est naturel de se défendre ", ou encore «le professeur Levi-Strauss m’a jeté le gant; je le relève ». Cette réaction s'explique en partie par des difficultés de compréhension mutuelle. Ainsi, le «formalisme » dont Levi-Strauss parle dans son article n'a pas le même contenu que pour Propp : pour le premier, ce n'est qu'une des méthodologies possibles d'analyse du texte littéraire tandis que pour le deuxième, accusé avec beaucoup d'autres de formalisme par les pouvoirs de la science littéraire soviétique de l'époque, il s'agit là d'une accusation idéologique. Ce décalage idéologique, lié à une compréhension

I4. S. J. Lourier, «Dom v lésou » (La maison dans la forêt), dans Jazyk i literatura (La langue et la littérature), Leningrad, I932, vol. VIII, p. I59-I94.

15. On utilise ce terme dans le sens d'Aarne et Thompson, voir infra.

I6. Voir, par exemple, l'article de R. L. Erlich "Skazka o lovkom vore" (Le conte du voleur adroit), ibid., p. 195-2004.

17. S. Thompson, Motif - Index of Folk Litterature, vol. I-6, Bloomington, I955-1958.

18. C. Lévi-Strauss, "La structure et la forme. Réflexions sur un ouvrage de Vladimir Propp ", Anthropologie Structurale, II, Paris, Plon, 1973.

I9. V. Ja. Propp, «Structurnoe i istoricheskoje izouchenie volchebnoj skazki », dans son Folklor i dejstvitel'nost' (Folklore et réalité), Moscou, 1976. Publication en italien : V. Ja. Propp, Morfologia della fiaba. Con un intervento di Claude Lévi-Strauss et una replica dell'autore. A cura di Gian Luigi Bravo, Torino, 1966, p. 20I-227. 
du texte français insuffisante, produit parfois des effets impressionnants : ainsi une expression aussi neutre et innocente que la "vision formaliste " dont parle Levi-Strauss se transforme, dans la traduction de Propp, en "fantôme formaliste ", formule assez dépréciative; de même, l' " obsession des explications historiques » devient "l'horrible nécessité des explications historiques ".

La réaction de Propp à l'article de Levi-Strauss rend difficile la collaboration entre les successeurs des formalistes en Union soviétique et les structuralistes français, bien que les premiers aient fourni aux seconds un matériel indispensable. L'approche structuraliste, qu'elle soit pratiquée par un chercheur soviétique ou par un étranger, est en général mal vue par les autorités idéologiques de la science des lettres en Union soviétique, pendant les années 1960-1980, tout comme l'était le formalisme peu de temps avant. Un des premiers structuralistes dont les ouvrages voient le jour à l'époque soviétique est E. M. Mélétinski (1918-2005) ${ }^{20}$ dont l'article "L'étude structurale et typologique du conte " parait en 1973, dans l'édition de Morphologie du conte ${ }^{21}$. Nous reviendrons ci-dessous sur les ouvrages que Mélétinski et ses élèves ont consacrés au conte, études aussi bien historiques que structurales.

L'époque où paraît la Morphologie du conte, c'est-à-dire fin des années I920 - début des années I930, est encore relativement favorable aux études de structure narrative. L'année suivante, après la Morphologie du conte, voit le jour l'Index des contes selon le système d'Aarne, de N.P. Andreev, collègue de Propp et de Nikiforov dans la Commission du conte. On se rappelle que dans sa Morphologie du conte comme dans ses articles, Propp critique l'ouvrage d'Aarne pour son explication inexacte de la notion de sujet ou de type, ainsi que pour l'impossibilité de bien distinguer certains types (de sorte qu'on peut parfois référer le même conte à cinq types à la fois $\left.{ }^{22}\right)$. Andreev applique toutefois le système d'Aarne aux matériaux du conte russe. Cinquante ans plus tard, verra le jour l'Index comparatif des contes types: Contes des Slaves de l'Est, de L. Barag, I. Berezovskij, K. Kabasnikov et N. Novikov, prenant pour point de départ l'index d'Andreev. Une édition universitaire de contes slaves est actuellement inimaginable sans références à cet index ${ }^{23}$.

20. Eleasar, et non Euguéni, comme il est faussement nommé dans l'édition de Propp ci-dessus mentionnée.

2I. V.Ja. Propp, Morphologie du conte, Paris, Seuil, 1973.

22. Ibid., p. 20.

23. L. G. Barag (éd.), I.P. Berezovskij, K. Kabashnikov, N. Novikov, Sravnitel'nyj ukazatel' suzetov. Vostochno-slavjanskaja skazka (Index comparatif des contes-types : contes des Slaves de 
En 1967, Viktor Zirmunskij, en évoquant dans son article «À propos des conte-types internationaux ${ }^{24}$ ", la proximité thématique de ces contes, souligne ensuite que cette proximité n'est pas totale, et qu'elle est limitée par les frontières d'une zone culturelle donnée. En Indonésie notamment, on peut marquer la frontière après laquelle les types systématisés par Aarne et Thompson s'épuisent, et où dominent les types ignorés par ce catalogue. En effet, le catalogue d'Aarne et Thompson, fait, avant tout, pour le conte européen ou "indo-européen ", n'embrasse pas toute la diversité thématique du conte dans d'autres aires géographiques et culturelles, et voilà la raison pourquoi notamment W. Eberhard ne put appliquer le système d'Aarne et Thompson au matériel chinois et fut obligé d'élaborer un nouveau catalogue selon une autre méthode ${ }^{25}$. Zirmunskij évoque la nécessité de l'élaboration d'un nouveau catalogue universel des types de contes, appuyé sur une nouvelle méthodologie plus rationnelle.

Nous nous permettons de mentionner ici encore un ouvrage de $\mathrm{N}$. Andreev : sa préface et ses commentaires à l'édition des contes de Charles Perrault ${ }^{26}$. Dans les commentaires, Andreev tient compte de toutes les éditions parues en français et des ouvrages fondamentaux consacrés à ces contes à l'époque, comme par exemple le livre de Saintyves ${ }^{27}$. Les contes de Perrault sont édités en 1934 dans une traduction fine et fidèle réalisée par Serguei Bobrov, poète bien connu du début du $\mathrm{xx}^{\mathrm{e}}$ siècle et chef du groupement poétique de futuristes nommé « Centrifuge » (Tsentrifuga). Cependant son nom n'apparaît pas dans l'édition du volume Perrault, car au moment où cette dernière voit le jour, Bobrov est détenu dans un camp stalinien.

l'Est), Leningrad, Naouka, 1979. Cet index ne tient pas compte des ajouts apportés à la classification d'Aarne faits par Stith Thompson. Actuellement, dans les études sur le conte slave, folklorique ou littéraire, on se réfère souvent à deux index - celui de Barag et celui d'Aarne-Thompson. De ce dernier, les chercheurs actuels préferent de plus en plus la version récente, comportant les ajouts de H.-J. Uther. Or, Heda Jason, dans son compte-rendu du dernier ouvrage, déconseille de dénoncer trop vite celui d'Aarne-Thompson : H. Jason, « Review of Uther H.-J., The Types of International Folktales ", Fabula, vol. 47, 2006, p. 172-186.

24. V.M. Zirmunskij, "À propos des conte-types internationaux» (Kvoprosou o mejdounaronhyh skazotchnyh sujetah) dans V. M. Zirmunskij, Le folklore de l'Ouest et de l'Est (Folklor zapada i vostoka), Moscou, OGI, 2004, p. 435-444.

25. W. Eberhard, Typen chinesicher Volkmärchen, Helsinki, 1937.

26. Charles Perrault, Skazki, Leningrad, Academia, 1936.

27. P. Saintyves, Les contes de Perrault et les récits parallèles. Leurs origines (coutumes primitives et liturgies populaires), Paris, E. Nourry, 1923, rééd. Bouquins, 1987. 
Les rapports entre le conte populaire et la littérature russe aux XviII$\mathrm{XIX}^{\mathrm{e}}$ siècles ont été étudiés par M. K. Azadovskij, dans Le Folklore et la littérature $^{28}$, et ensuite par E. V. Pomerantseva, dans Destins du conte russe ${ }^{29}$. Marc Azadovskij concentre ses études autour des emprunts folkloriques dans la littérature de la première partie du $\mathrm{XIX}^{\mathrm{e}}$ siècle et des travaux des folkloristes de la même époque, dans tous les genres de folklore. Il ne limite pas sa recherche au matériel russe de la tradition orale, mais il étudie également les motifs du conte populaire introduits dans la littérature russe à travers des sources variables, telles que la collection de contes publiée par les frères Grimm. L'objet de son intérêt le plus profond, dans le champ littéraire russe du début du XIx ${ }^{e}$ siècle, est constitué par les contes de Pouchkine. Quant à Erna Pomerantseva (Hoffmann), elle étudie le genre du conte littéraire aux XVIII ${ }^{\mathrm{e}}$-XIX ${ }^{\mathrm{e}}$ siècles, et les rapports entre le conte littéraire et folklorique. Erna Pomerantseva est une folkloriste remarquable du siècle précédent : sa collection de chansons et de romances urbaines est d'une richesse impressionnante ; elle est également l'auteur d'une grande quantité d'études sur le conte populaire : il faut signaler spécialement son article consacré au style individuel du conteur de contes populaires ${ }^{30}$, où elle précise l'importance du classement de contes folkloriques dans le recueil par conteurs, car leur manière de conter se manifeste indépendamment des caractéristiques génériques de chaque texte que ces derniers articulent.

Parmi les études ethnographiques du conte oral, il faut remarquer celle de D. Zelenin ${ }^{31}$. Celui-ci parle de la valeur magique des contes; il décrit la situation sémiotique dans laquelle les contes archaïques et les récits mythologiques étaient racontés. C'était essentiellement la situation de la chasse, où l'on mimait les animaux, leur racontant des histoires ou des contes pour les attirer. Plus tard, ce furent les démons-patrons des animaux à qui les chasseurs destinaient leurs récits pour obtenir la proie en récompense. Ainsi le conte est historiquement et pragmatiquement lié aux formules magiques, et il fait partie des rites "primitifs". Zelenin parle également des tabous relatifs au fait de conter contes et récits mythologiques, d'après

28. M. K. Azadovskij, Folklor i literatura, Leningrad, 1938.

29. E. V. Pomerantseva, Sout'by rousskoj skazki, Moscou, 1965.

30. E. V. Hoffmann, «K voprosu ob individualnom stile skazochnika » (À propos du style individuel du conteur), Houdozestvennyj folklor, Moscou, vol. IV-V, I929, p. II3-II9.

3I. D. K. Zelenin, articles des 1934-1954, Moscou, Indrik, 2004, p. 19-44.

Pour la première version de cet ouvrage, en Allemagne, voir D. Zelenin, « Die religiöse Funktion der Volkmärchen ", dans Internationales Archiv für Ethnographie, vol. XXXII, 1932 [19IO], p. 2I-3I. 
les croyances bien répandues en de nombreuses régions, d'après lesquelles cela pouvait nuire aux troupeaux.

Nous avons mentionné ci-dessus la monographie de V.D. Kuz’mina consacrée aux origines de certains contes loubok russes. La tradition du livre de colportage en Russie n'est que très peu étudiée : K. E. Korepova travaille actuellement sur la tradition de loubok et ses liens avec les premiers recueils de contes ${ }^{32}$, en étudiant l'influence réciproque des traditions du conte littéraire, conte de colportage et conte oral, et elle décrit le mécanisme de cette influence et du passage d'un texte de la tradition livresque (notamment les Contes Russes de Levchine) vers la tradition orale. Elle montre comment certaines histoires que Levchine puise dans la tradition populaire sont reprises par les éditeurs de loubok, et comment ensuite, à travers les livres de colportage lus et racontés dans les milieux populaires, ils reviennent dans la tradition orale. Dans le tableau proposé ci-dessous, nous voyons comment un conte paru sous forme du loubok change en passant ensuite vers la tradition orale. Korepova étudie ici un conte de loubok se trouvant dans la collection de Rovinskiy ${ }^{33}$, et ses versions orales rassemblées par Houdiakov ${ }^{34}$ et par Nikiforov ${ }^{35}$. Dans la colonne I du tableau ci-dessous, nous voyons le mot « палица " [palitsa = la massue], qui, vers la fin du XIX ${ }^{e}$ siècle, est ressenti comme suranné et « trop littéraire ». À force d'être peu compréhensible de l'auditoire populaire illettré, ce mot est transmis par les conteurs de deux manières différentes et toujours fautives. Dans le recueil de Houdiakov (la colonne 2), l'origine du malentendu est phonétique : l'incompréhensible [palitsa], la massue, se transforme en objet plus connu, mais phonétiquement semblable - [palits] le doigt, se précisant ensuite en « auriculaire ». Dans le livre de Nikiforov (colonne 3), la transformation est purement sémantique. Le mot "massue " [palitsa], dans ce cas-là bien compris par le conteur, mais visiblement exclu de son vocabulaire actif, se transforme en bâtonnet, objet beaucoup plus familier.

32. K. E. Korepova, Rousskaja loubochnaja skazka (Le conte loubok russe), Nijni Novgorod, 1999. La deuxième rédaction complétée de ce livre est à paraitre ultérieurement.

33. D. A. Rovinskiy, Rousskie narodnyje kartinki (Tableaux populaires russes), 5 vol., SaintPétersbourg, I88I.

34. I. A. Houdiakov, Velikorousskije skazki v zapisjah Houdjakova, Moscou, Leningrad, 1964.

35. A. I. Nikiforov, Severnorousskije skazki v zapisjah Nikiforova. Texte preparé par V. Propp, Moscou, Leningrad, I96r. 


\begin{tabular}{|c|c|c|}
\hline $\begin{array}{l}\text { I. Loubok (livre populaire), } \\
\text { collection de Rovinskiy }\end{array}$ & $\begin{array}{l}\text { 2. Conte oral - I, collection } \\
\underline{\text { de Houdiakov }}\end{array}$ & $\begin{array}{l}\text { 3. Conte oral -2, collection } \\
\text { de Nikiforov }\end{array}$ \\
\hline \multicolumn{3}{|c|}{$\begin{array}{l}\text { Pour se déplacer vers l'autre royaume, le héros s'accroche à l'objet magique proposé } \\
\text { par son adjoint. }\end{array}$} \\
\hline \multicolumn{3}{|l|}{ L'adjoint } \\
\hline Le Tourbillon (Le Géant) & Le Tourbillon (Le Géant) & L'Oiseau gigantesque \\
\hline \multicolumn{3}{|l|}{ L'objet magique } \\
\hline $\begin{array}{l}\text { Палица }[\text { palitsa] }= \\
\text { la massue }\end{array}$ & $\begin{array}{l}\text { Мизинец [mizinets] = } \\
\text { *палец [palits] = } \\
\text { l'auriculaire (le doigt) } \\
\text { phonétique } \rightarrow \text { sémantique }\end{array}$ & $\begin{array}{l}\text { Костылек [kostyljok] = } \\
\text { le bâtonnet; sémantique }\end{array}$ \\
\hline
\end{tabular}

Fig. I. - Mécanisme du passage de la tradition livresque vers la tradition orale.

Les mêmes mécanismes de transformation et d'altération du texte se trouvent à la frontière de la culture livresque et de l'oralité.

L'approche à la fois structurelle et historique est pratiquée par E. M. Mélétinski (déjà mentionné ci-dessus) et par son école, dans leurs études de la narration mythologique, folklorique et littéraire. Dans son ouvrage monographique Le Héros du conte merveilleux $x^{36}$, Mélétinski observe l'histoire de certains héros les plus typiques du conte merveilleux, à commencer par le mythe étiologique et le conte archaïque ou mythologique, jusqu'au conte merveilleux de la nouvelle époque. Il s'agit des héros humiliés ou méprisés, tels que le pauvre orphelin, le frère cadet, la belle-fille persécutée et l'humble héros du conte. La formation du type de protagoniste est un produit des processus socio-historiques, voilà pourquoi, dans son étude, Mélétinski s'applique au folklore des peuples dont les niveaux de développement social sont différents et dont le folklore fournit ainsi une espèce de champ d'expérience. Ce livre comparatiste dont l'approche typologique et structurale n'a pas été bien accueillie par la science soviétique en général, n'aurait peut-être pas vu le jour si son auteur n'avait présenté les héros humbles et persécutés du conte comme des "idéaux démocratiques »- ruse innocente qui lui servit de sauf-conduit dans la

36. E. M. Mélétinski, Geroj volchebnoj skazki, Moscou, 1958. 
science des lettres soviétiques. Son autre livre bien connu, La poétique du mythe, porte sur les particularités de la conscience mythologique et sur les liaisons structurelles, historiques et sémantiques entre le mythe, le conte et l'épopée. La dernière partie de l'ouvrage est consacrée au mythologisme dans la fiction du $\mathrm{xx}^{\mathrm{e}}$ siècle. La poétique du mythe est traduite en dix langues dont l'anglais, l'italien, le portugais et le chinois. Eléazar Mélétinski travaille également sur la poétique historique du folklore narratif (conte, épopée) et de la littérature narrative (roman, nouvelle).

Les années 1960-1970 marquent l'époque de la naissance du structuralisme et de la sémiotique russes. Sans jamais favoriser ou approuver ces deux écoles, les pouvoirs de la science soviétique feignaient de ne pas les remarquer. À l'université de Tartou (Estonie), l'école sémiotique était dirigée par Ju. M. Lotman, l'hérétique reconnu de la science littéraire ; l'autre partie de l'école sémiotique, plus orientée vers la linguistique, se trouvait à Moscou. C'est également à Moscou qu'à la fin des années 1960, le groupe d'études de la structure du conte merveilleux dirigé par E.M. Mélétinski commence son travail. Les autres membres de ce collectif sont S. Ju. Nekljudov, E. S. Novik et D. M. Segal. Faute de permission de la direction, ce groupe rassemblant essentiellement les chercheurs de l'Institut de la littérature mondiale ne peut pas être affilié à l'Institut et se réunit dans la maison d'E. M. Mélétinski, presque clandestinement.

Dans leur ouvrage monographique La structure du conte merveilleux, E. M. Mélétinski, S. Ju. Nekljudov, E. S. Novik et D.M. Segal utilisent les résultats de la recherche de Propp, des structuralistes français (tels que $\mathrm{C}$. Levi-Strauss, A. J. Greimas et C. Bremond) et des linguistes russes dont tout d'abord V.N. Toporov et V.Vs. Ivanov. Ils révèlent, en précisant le modèle de Greimas, les blocs binaires de fonctions et les appliquent au système d'actants (par exemple, dans le bloc "demande - exécution ", la non-exécution de la demande du donateur mène au résultat négatif et la non-exécution de la demande de l'agresseur au résultat positif). Ainsi toutes les fonctions dans le conte forment des blocs binaires. Grâce à ce principe binaire, chaque épisode a une composition symétrique (départ - retour, ordre - exécution, tâche - résolution). Le principe binaire (adjuvant - opposant, interne - externe, familier - étranger) est utilisable pour décrire le système de personnages, mais également pour caractériser l'espace et le temps dans le conte merveilleux. En analysant la composition et la structure des relations de personnages dans le conte merveilleux, les auteurs de la monographie prennent en compte non seulement les blocs binaires, mais aussi les triades typiques pour ce genre du folklore. Ils 
appliquent à la structure du conte la formule de médiation élaborée par Levi-Strauss pour la structure mythologique.

En 1969 et 197I, des parties de cette monographie paraissent dans les Actes de l'université de Tartu Troudy po znakovym systemam ("Les mémoires des systèmes significatifs"). En 1971, l'ouvrage obtient le prix international de Pitrè ; en 1974, il est traduit et publié en anglais, au 1977 en italien, en 1986 en allemand, et en 1992 en français ${ }^{37}$. Le départ de D. Segal à l'étranger rend impossible non seulement la poursuite du travail, mais aussi la publication de la monographie en Union Soviétique. Cet ouvrage ne paraît en Russie qu'en $200{ }^{38}$. Y sont rassemblés sous une même reliure l'article d'E. S. Novic « Le système de personnages du conte merveilleux russe », ainsi que celui d'E. M. Mélétinski "Étude structurale et typologique du conte " mentionné ci-dessus. L'édition de $200 \mathrm{I}$ inclut également l'article panoramique d'A. Rafaeva, E. Rakhimova et A.S. Arkhipova sur "L'étude structurelle et sémiotique du conte au $\mathrm{Xx}^{\mathrm{e}}$ siècle, en Russie et à l'étranger ", où il est également question des logiciels de création de contes.

Il reste à mentionner les ouvrages les plus récents consacrés à l'étude du conte. Les thèmes, les structures et les motifs des contes et des mythes attirent l'intérêt des spécialistes russes. Actuellement, plusieurs nouveaux catalogues de sujets du conte et des autres genres du folklore narratif paraissent sous forme livresque et sont également disponibles, informatisés, sur le site internet <http://www.ruthenia.ru/folklore/indexes.htm>, consacré aux problèmes de structure, de typologie et de sémiotique du folklore et du postfolklore (terme proposé par S. Ju. Nekljudov pour définir le folk-

37. E. Meletinsky, S. Nekludov, E. Novik, D. Segal, «Problems of the Stuctural Analysis of Fairytales ", Soviet Structural Folkloristics. Texts by Meletinsky, Nekludov, Novik, and Segal with Tests of the Approach by Jilek and Jilek-Aall, Reid and Layton. Introduced and ed. P. Maranda. Vol. I, The Hague-Paris, 1974, p. 73-I39;

E. M. Mélétinskij, S. Ju. Nekljudov, E.S. Novik, D. Segal, La struttura della fiaba, Palermo, Sellerio editore, 1977, p. 54-I37.

E. M. Mélétinskij, S. Ju. Nekljudov, E. S. Novik, D. Segal, «Probleme der strukturalen Beschriebung des aubermaerchens. Noch einmal zur Problem der strukturalen Beschriebung des Zaubermaerchens ", dans Semiotica Sovietica. Sowijetische Arbeiten der Moskauer und Tartuer Schule zu sekundaeren modellbildenden Zeichensystemen (1962-1973). Hrsg. u. eingel. von K. Eimermacher. Aachen, "Rader-Verlag », 1986 (Aachener Studien zur Semiotik und Kommunikationsforschung. Bd 5), p. 199-318.

E. Meletinsky, S. Neklioudov, E. Novik, D. Segal, «Problèmes de la description structurale du conte merveilleux. Retour sur le problème de la description structurale du conte merveilleux ", dans J. Letourneau (éd.), Travaux de sémiotique narrative, Université Laval, Rapports et Mémoires de recherches du Celat, nº 2I, Québec, I992, p. I-82.

38. Strouktoura volchebnoj skazki (La structure du conte merveilleux), Moscou, 200I. 
lore contemporain et essentiellement urbain). Ce projet informatique est dirigé par Nekljudov et son équipe. Dans cette dernière partie de notre panorama, nous nous référerons souvent à ce site, car pour les études folkloriques en Russie, c'est aujourd'hui la ressource la plus riche : tous les catalogues de types et de motifs du folklore narratif russe disponibles aujourd'hui s'y trouvent, ainsi que des matériaux qui dépassent les marges propres du folklore russe.

La spécialiste lituanienne Bronislava Kerbelite propose son propre principe de catalogage des sujets de contes et légendes, alternatif à celui d'Aarne et Thompson. Sur le site <http://www.ruthenia.ru/folklore/kerbeliter.html>, on peut trouver son catalogue de types de légendes étiologiques, mythologiques et historiques de Lithuanie. B. Kerbelite propose de prendre en compte les éléments mineurs de la narration, tels que les "sujets minimaux ", car les types de contes se mêlent souvent. Cet ouvrage est également disponible en anglais.

Ju.E. Berezkin propose sa classification thématique et son schéma de distribution mondiale des motifs folkloriques et mythologiques, sur le site <http://www.ruthenia.ru/folklore/berezkin/index.htm>.

Parmi les autres genres narratifs pris en compte par les auteurs des catalogues de sujets, se rencontrent les bylina, un genre, ne l'oublions pas, tout à fait essentiel pour les auteurs des premiers contes littéraires en Russie. La base de données recensant les sujets des bylina, élaborée par N.V. Petrov, est disponible sur le site <http://www.ruthenia.ru/folklore/petrov/index. htm>.

Récemment A.V. Kozmin, A. S. Arkhipova et A. Rafaeva ont élaboré le système informatique d'analyse du conte au niveau de la structure "médiator " ou "skazka" (conte). Ce système emploie les contes-types d'Aarne - Thompson et le Motif index de S. Thompson ; il prend en compte les fonctions de Propp et les actants de Greimas, pour analyser les sujets des contes de l'édition d'Afanssiev. Le système «skazka " permet de trouver un sujet de conte selon son numéro dans le catalogue ou selon les critères donnés par l'utilisateur. Cet outil est encore en cours d'élaboration, mais déjà partiellement disponible sur <http://www.ruthenia.ru/ folklore/kozmin5.htm>, et <http://www.ruthenia.ru/folklore/xmlfabula/ test6.htm>.

Si les sujets des genres narratifs folkloriques, avec leur structure relativement stricte, permettent de les cataloguer avec une précision remarquable, ce n'est pas le cas de la narration littéraire dont la variété de tous les " possibles narratifs" est beaucoup plus vaste. Toutefois, plusieurs essais 
dans cette direction ont été entrepris récemment. Ainsi T. A. Kitanina est actuellement en train de travailler sur le catalogue structurel des sujets littéraires. Certains matériaux de ce catalogue sont déjà publiés, bien que ce travail énorme soit encore loin d'être achevé.

L'interaction entre la narration orale et littéraire est jusqu'à aujourd'hui relativement peu étudiée par la science des lettres en Russie, faute de collaboration suffisante entre les spécialistes de la littérature et du folklore. Néanmoins, plusieurs ouvrages collectifs où sont, entre autres, recueillis des articles consacrés aux narrations orales et littéraires, viennent de paraître ou sont à paraitre très prochainement ${ }^{39}$. Espérons que cela ouvrira une féconde époque de recherches en ce domaine.

39. Kirpichiki ("Les petits briques») : Les études folkloriques et antropologiques aujourd'hui. Recueil des articles consacré au $65^{\mathrm{e}}$ anniversaire de $S$. Ju. Nekljudov, et au $40^{\mathrm{e}}$ anniversaire de son activité scientifique ; Moscou, RGGU, 2008. Slovo ustnoe i slovo knijnoye ("Parole orale, parole littéraire ", ouvrage collectif, consacré à l'intéraction de la tradition orale et de la tradition littéraire), à paraître, Moscou, RGGU, 2009. 